University of Wollongong

Research Online

Faculty of Informatics - Papers (Archive)

Faculty of Engineering and Information

Sciences

2006

\title{
Genetic analysis of weight, fat and muscle depth in growing lambs using random regression models
}

T. Fischer

University of New England

J. van der Werf

University of New England

R. Banks

University of New England

A. Ball

University of New England

Arthur R. Gilmour

Orange Agricultural Institute, agilmour@uow.edu.au

Follow this and additional works at: https://ro.uow.edu.au/infopapers

Part of the Physical Sciences and Mathematics Commons

\section{Recommended Citation}

Fischer, T.; van der Werf, J.; Banks, R.; Ball, A.; and Gilmour, Arthur R.: Genetic analysis of weight, fat and muscle depth in growing lambs using random regression models 2006.

https://ro.uow.edu.au/infopapers/3773

Research Online is the open access institutional repository for the University of Wollongong. For further information contact the UOW Library: research-pubs@uow.edu.au 


\title{
Genetic analysis of weight, fat and muscle depth in growing lambs using random regression models
}

\begin{abstract}
Genetic parameters were estimated using uni- and bi-variate random regression models for weight, eyemuscle depth and fat depth measures between 60 and 360 days of age. Each trait was measured up to five times in 50-day intervals following weaning on approximately 4000 Australian Poll Dorset Sheep. The model accounted for rearing type, dam age, management group and age of recording. The model used for analysing weight included quadratic, orthogonal polynomials for direct genetic and environmental effects, a linear polynomial for maternal genetic effects and heterogeneous error variance across ages. The fat and muscle analysis used linear orthogonal polynomials for direct genetic and environmental effects and heterogeneous error variance. Throughout the 300-day trajectory heritability for weight traits ranged from 0.20 to 0.31 , while heritability for fat depth ranged from 0.24 to 0.34 and heritability for eyemuscle depth ranged from 0.24 to 0.40 . Genetic correlations between repeated measures of the same trait at different ages were positive and declined as the age interval increased, to minimum values of $0.60,0.31$ and 0.50 for weight, fat and muscle respectively between 60 and 360 days of age. Genetic correlations between weight and fat and weight and eye muscle were moderate to high (0.6 to 0.8$)$ and positive but decreased slightly with age. The genetic correlations between fat and muscle were moderate to high $(0.5$ to 0.7$)$ throughout the 300-day trajectory. In all cases, the estimates produced in this study were reasonably consistent with the limited number of studies that exist in the reported literature. This study demonstrated the relationships that exist between repeated measures of weight, fat and muscle measures over time, which is of interest to prime lamb producers looking to select for specific breeding objectives or market end points requiring precise weight, fat and muscle combinations at certain ages.

\section{Disciplines}

Physical Sciences and Mathematics

\section{Publication Details}

Fischer, T. M., van der Werf, J. H. J., Banks, R., Ball, A. J. \& Gilmour, A. R. (2006). Genetic analysis of weight, fat and muscle depth in growing lambs using random regression models. Animal Science, 82 (1), 13-22.
\end{abstract}




\title{
Genetic analysis of weight, fat and muscle depth in growing lambs using random regression models
}

\author{
T. M. Fischer ${ }^{1,4,5 \dagger}$, J. H. J. van der Werf ${ }^{1,4}$, R. G. Banks ${ }^{2}$, A. J. Ball ${ }^{2}$ and A. R. Gilmour ${ }^{3,4}$ \\ ${ }^{1}$ School of Rural Science and Agriculture, University of New England, Armidale, NSW 2351, Australia \\ ${ }^{2}$ LAMBPLAN, MLA, c/o Animal Science, UNE, Armidale, NSW 2351, Australia \\ ${ }^{3}$ NSW Agriculture, Orange Agricultural Institute, Orange, NSW 2800, Australia \\ ${ }^{4}$ Australian Sheep Industry CRC \\ ${ }^{5}$ Australian Wool Innovation, 16-20 Barrack Street, Sydney, NSW 2000, Australia
}

${ }^{\dagger}$ Correspondence address: Australian Wool Innovation, 16-20 Barrack Street, Sydney, NSW, 2000, Australia. E-mail: troyfischer@woolinnovation.com.au

\begin{abstract}
Genetic parameters were estimated using uni- and bi-variate random regression models for weight, eye-muscle depth and fat depth measures between 60 and 360 days of age. Each trait was measured up to five times in 50-day intervals following weaning on approximately 4000 Australian Poll Dorset Sheep. The model accounted for rearing type, dam age, management group and age of recording. The model used for analysing weight included quadratic, orthogonal polynomials for direct genetic and environmental effects, a linear polynomial for maternal genetic effects and heterogeneous error variance across ages. The fat and muscle analysis used linear orthogonal polynomials for direct genetic and environmental effects and heterogeneous error variance. Throughout the 300-day trajectory heritability for weight traits ranged from 0.20 to 0.31 , while heritability for fat depth ranged from 0.24 to 0.34 and heritability for eyemuscle depth ranged from 0.24 to 0.40 . Genetic correlations between repeated measures of the same trait at different ages were positive and declined as the age interval increased, to minimum values of $0.60,0.31$ and 0.50 for weight, fat and muscle respectively between 60 and 360 days of age. Genetic correlations between weight and fat and weight and eye muscle were moderate to high (0.6 to 0.8) and positive but decreased slightly with age. The genetic correlations between fat and muscle were moderate to high (0.5 to 0.7) throughout the 300-day trajectory. In all cases, the estimates produced in this study were reasonably consistent with the limited number of studies that exist in the reported literature. This study demonstrated the relationships that exist between repeated measures of weight, fat and muscle measures over time, which is of interest to prime lamb producers looking to select for specific breeding objectives or market end points requiring precise weight, fat and muscle combinations at certain ages.
\end{abstract}

Keywords: genetic parameters, growth, regression analysis, sheep.

\section{Introduction}

Precise description of growth and development is becoming more important in highly productive meat and livestock industries around the world (e.g. the Australian lamb industry). Meat processors are becoming more focussed on specific products that meet tight specifications with the ultimate aim of supplying the consumer with a consistently high quality product. In addition, different markets have specific requirements, hence knowledge on the genetics of growth and development of individual animals that are suited to particular markets is of immense interest to such industries. To this end, livestock producers need accurate genetic information on which to base their selection and management decisions so that their animals meet these specifications. In order to achieve the breeding objectives of such lamb producers, that is, lambs that grow heavy, lean, muscular carcasses in a short amount of time, we need to know the relationships between these traits, and in particular how these relationships change with age.

Moreover, there is interest in selection of animals to alter the average growth curve of the population to improve efficiency of the production system. The desire to overcome the genetic antagonism between rapid, efficient early growth of slaughter progeny and smaller size and lower maintenance cost of parental stocks remains (Kinghorn, 1997). The same efficiency principle can also be applied to carcass composition traits (e.g. the optimal moment of onset of fat deposition), and this is perhaps more important than weight per se, as fat is three or four times more energetically expensive to deposit than muscle (Thompson and Butterfield, 1985). Hence, it is of interest to have a more precise description of genetic and phenotypic variation of weight, fat and muscle traits with respect to age. 
Development of effective genetic improvement programs requires knowledge of the genetic variation for economically important traits and the genetic covariance between those traits. In Australia, the national genetic evaluation program, LAMBPLAN, has been providing estimated breeding values for weight, fat and muscle depth at fixed points in time (e.g. 200 days) since 1989 (Banks, 1990). Genetic parameters used for the LAMBPLAN evaluation have been based on a review by Fogarty (1995) and those estimated by van der Werf and Wheaton (1999). However, the relationships among these traits are best known at post weaning age (around 200 days) and there is little known about them at other ages. To investigate this, repeated measurements of weight, fat and muscle depth were obtained for this study from an Australian meat sheep (Poll Dorset) breeding nucleus, Meat Elite (Banks, 1997). This data set was unique in the fact that a good number of animals (ca. 4000) had repeated measures of weight, fat and muscle depth and that these animals originated from large, well recorded, genetically well linked flocks.

Random regression (RR) was chosen as the method for analysis of the growth and development traits in this study, as it has been identified as a useful way of computing breeding values for individual animals throughout the trajectory of interest. Application of RR models to growth data is reasonably well documented in all major livestock industries (e.g. Fischer et al. (2004), Huisman et al. (2002), Meyer (2002) and Veerkamp and Thompson (1999)), however, they have not been applied to carcass composition data with the exception of Hassen et al. (2003). In addition, multiple trait random regression (RR) models have been fitted between weight and other traits simultaneously (e.g. Huisman (2002) and Veerkamp and Thompson (1999)) but have not related weight with fat and muscle depth. This is largely due to the scarcity of suitable data, that is, repeated measures of carcass traits on individual animals. Due to the high costs of obtaining this data, i.e. carcass data via ultrasound, repeated measures of such data are generally not collected in industry practice.

The aim of this study was to estimate genetic parameters for weight, fat and muscle measures when treated as continuous functions of age using uni- and bi-variate random regression models. This includes genetic and phenotypic correlations within and between the three traits throughout a 300-day trajectory. The potential for breeders to exploit this information when making selection decisions will be discussed.

\section{Material and methods}

Data

Data was collected in the period 2002 to 2003 from 15 Poll Dorset sheep flocks which comprise the current Meat Elite nucleus breeding scheme in Australia (Banks, 1997), and consisted of repeated weight, fat and eye-muscle depth records on all 2002 drop animals (ca. 4000) in those flocks. Body weight (BW), ultrasonic fat depth (FD) and ultrasonic eye-muscle depth (EMD) at the $C$ site of the 12/13th rib were collected three to five times in 50-day intervals between 150 and 350 days of age. Accredited operators scanned all animals in each flock. In addition, data on the parents of the 2002-drop animals plus their contemporaries were included to account for selection leading up to the production of this drop of animals, as well as to enable estimation of maternal effects for weight traits. This extended the data set by around $100 \%$ for the BW data and $50 \%$ for the FD and EMD data.

Standard data edits excluded animals without a recorded Poll Dorset sire and dam, contemporary groups of less than 10 animals were omitted and only records within a specified age range (60 to 360 days) were included. This age range was chosen because there were very few records at ages younger than 60 days or at ages beyond 360 days. Detailed characteristics of the data structure are given in Table 1.

In addition to these edits, only animals with at least one weight, fat and muscle measure at the same age were used in the bivariate analysis in the interests of reducing the size of the analysis and removing non-informative animals (e.g. animals with one record for one trait). This yielded a total of 28239 weight, 19322 fat depth and 19675 eye-muscle depth records on 10911 animals. Consequently, there was an average of 1.8 weight, fat and muscle triplicates per animal. Animals with records were progeny of 471 sires, 6745 dams and 748 maternal grand-sires. Pedigree for parents was obtained for up to three generations back, resulting in a total of 19455 animals in the analysis.

Figure 1 shows the distribution of BW, FD and EMD measures with respect to age at measurement (10-day intervals). It shows there were more weight records at all ages in the trajectory relative to the carcass measures, especially at younger ages ( $<150$ days). The carcass measures were reasonably evenly distributed beyond 100 days.

$R R$ model used for analysis

Let $\mathrm{y}_{\mathrm{ijk}}$ denote the $j$ th record of the $k$ th trait for animal $i$ taken at age $t_{\mathrm{ijk}}$. The RR model is

$$
\begin{aligned}
\mathbf{y}_{\mathrm{ijk}}= & \mathrm{F}_{\mathrm{ijk}}+\sum_{\mathrm{m}=0}^{\mathrm{k}_{\mathrm{A}-1}} \boldsymbol{\alpha}_{\mathrm{im}} \boldsymbol{\phi}_{\mathrm{m}}\left(\mathbf{t}_{\mathrm{ijk}}\right)+\sum_{\mathrm{m}=0}^{\mathrm{k}_{\mathrm{B}-1}} \gamma_{\mathrm{im}} \boldsymbol{\phi}_{\mathrm{m}}\left(\mathrm{t}_{\mathrm{ijk}}\right) \\
& +\sum_{\mathrm{m}=0}^{\mathrm{k}_{\mathrm{C}-1}} \delta_{\mathrm{im}} \boldsymbol{\phi}_{\mathrm{m}}\left(\mathrm{t}_{\mathrm{ijk}}\right)+\varepsilon_{\mathrm{ijk}}
\end{aligned}
$$

with $t_{\mathrm{ijk}}$ denoting the age at recording for $y_{i j k}$, with $t$ standardised $(-1<t<1)$, and $\phi_{m}\left(t_{i j k}\right)$ the corresponding $m$ th Legendre polynomial values. $F_{i j k}$ represents the fixed effects

Table 1 Characteristics of the data structure

\begin{tabular}{lccc}
\hline \hline & Weight & Fat depth & Eye-muscle depth \\
\hline No. of animals & 17597 & 10869 & 10799 \\
\% of animals with one record & 13 & 35 & 34 \\
Sires & 542 & 464 & 459 \\
Dams & 8765 & 6848 & 6661 \\
Maternal grand sires & 820 & 741 & 749 \\
Animals in pedigree & 26166 & 19291 & 19210 \\
Records per animal (average) & $2 \cdot 7$ & $1 \cdot 8$ & 1.8 \\
Contemporary groups & 570 & 355 & 354 \\
\hline \hline
\end{tabular}




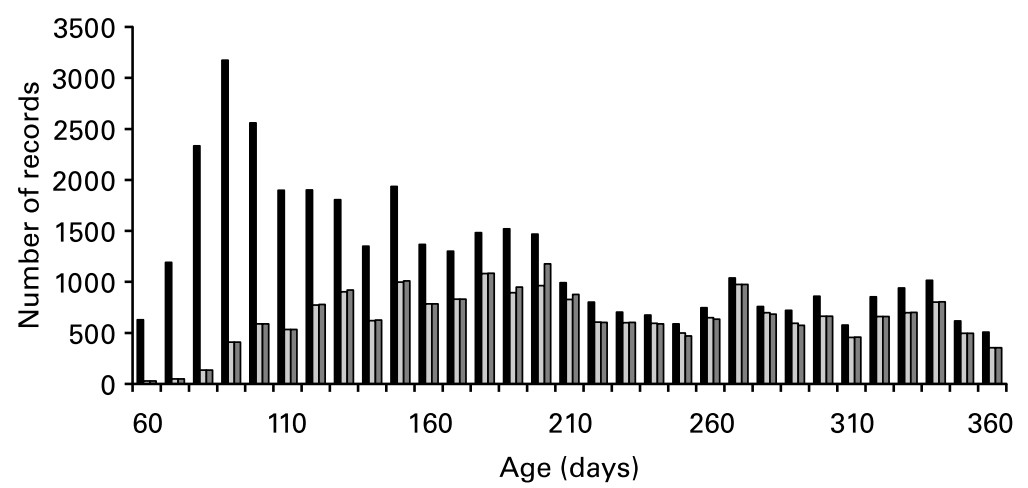

Figure 1 Numbers of weight (black bars), fat (light grey bars) and muscle (dark grey bars) records for individual ages in 10-day intervals.

pertaining to $y_{i j k}$. The variables $\alpha_{\mathrm{im}}, \delta_{\mathrm{im}}$ and $\gamma_{\mathrm{im}}$ are the $m$-th order RR coefficients for the direct additive genetic, maternal additive genetic and direct permanent environmental effects, respectively, and $\mathrm{k}_{\mathrm{A}-1}, \mathrm{k}_{\mathrm{B}-1}$ and $\mathrm{k}_{\mathrm{C}-1}$ are the corresponding order of (polynomial) fit for each effect. Finally, $\epsilon_{\mathrm{ijk}}$ denotes the residual error.

\section{Fixed effects}

Changes in the mean with age were modelled through a fixed regression using orthogonal polynomials of age (in days). Preliminary least-squares analyses ignoring animal effects were carried out using SAS' PROC GLM (Statistical Analysis Systems Institute, 1988) to determine the order of polynomial fit required to model the population trajectory. It was shown that orders of fit beyond quartic were non-significant for BW data and polynomials beyond quadratic were not significant for FD and EMD data. For the analysis of weight, a quartic Legendre polynomial of age was nested within levels of rearing type (single, twin or triplet) and dam age (in years - 12 classes) so that these fixed effect corrections varied across the trajectory. Similarly for fat and eye-muscle depth, a quadratic Legendre polynomial of age was nested within levels of rearing type. In addition, for BW, FD and EMD, contemporary groups (CG) were fitted as class variables unique to date of measurement with a small range in age for each group, hence, CG classes were defined by flock-year-paddock-measurement date-sex subclasses (570, 355 and 354 levels for BW, FD and EMD, respectively).

\section{Random effects}

All RR models used Legendre polynomials of age at recording (in days) as independent variables. The model for BW included quadratic polynomials for random components with the exception of maternal genetic effects, which were fitted using a linear polynomial. Models used for analysis of FD and EMD were the same as the models used for BW, except that a linear polynomial was used for the random regressions and maternal effects were excluded. Direct and maternal genetic effects were assumed to have a covariance structure proportional to the numerator relationship matrix. In the bivariate analysis, the covariance between traits for direct genetic and environmental effects was fitted as well. Residual effects for growth and carcass traits were modelled as a step function with 5 heterogeneous measurement error variance classes in 60-day intervals (e.g. 60$120, \ldots, 300-360$ days) and the covariance between residual effects for different traits was fitted in the bivariate analysis. All models were fitted using ASREML (Gilmour et al., 2002) which uses the average information algorithm to locate the maximum of the restricted likelihood (Gilmour et al., 1995).

\section{Covariance functions (CF)}

Parameters estimated in RR analyses were the $\mathbf{K}$ matrices containing variances and covariances between RR coefficients. The covariance function for any random effect (e.g. genetic effects defined by $\hat{G}_{\mathrm{o}}$ ) was obtained by pre and post multiplying $\mathbf{K}$ by a matrix containing Legendre polynomials $(\Phi)$ pertaining to a set of specific ages. The result is an estimated covariance matrix for that random effect for specific ages defined by $\boldsymbol{\Phi}$ (Kirkpatrick et al., 1990). For direct genetic effects for two traits, this can be shown in matrix notation as

$$
\hat{\mathbf{G}}_{\mathrm{o}}=\left[\begin{array}{cc}
\Phi_{1} \mathrm{~K}_{1} \Phi_{1}^{\prime} & \Phi_{1} K_{2,1}^{\prime} \Phi_{2}^{\prime} \\
\Phi_{2} K_{2,1} \Phi_{1}^{\prime} & \Phi_{2} K_{2} \Phi_{2}^{\prime}
\end{array}\right]
$$

where $\mathbf{K}_{1}$ is a $k_{1} \times k_{1}$ matrix containing the variances and covariances between random regression coefficients for trait 1 and $\phi_{1}$ is a $n \times k_{1}$ matrix that contains Legendre polynomials pertaining to a set of $n$ selected ages. Similarly, for trait 2 we have $K_{2}$, which is a $k_{2} \times k_{2}$ matrix, and $\phi_{2}$, which is a $n \times k_{2}$ matrix for another set of ages, however, the ages could be the same for $\phi_{1}$ and $\phi_{2}$. For the covariance between trait 1 and 2 we have $\mathbf{K}_{\mathbf{2}, \mathbf{1}}$, which is a matrix of dimension $k_{2} \times k_{1}$. These can then be used to calculate heritability and correlations within and between traits at various ages throughout the trajectory. In the results section, eigenvalue information, variance components, heritability estimates and correlations between measures of the same trait were derived from the univariate analyses and genetic and phenotypic correlations between different traits were derived from the bivariate analyses. 


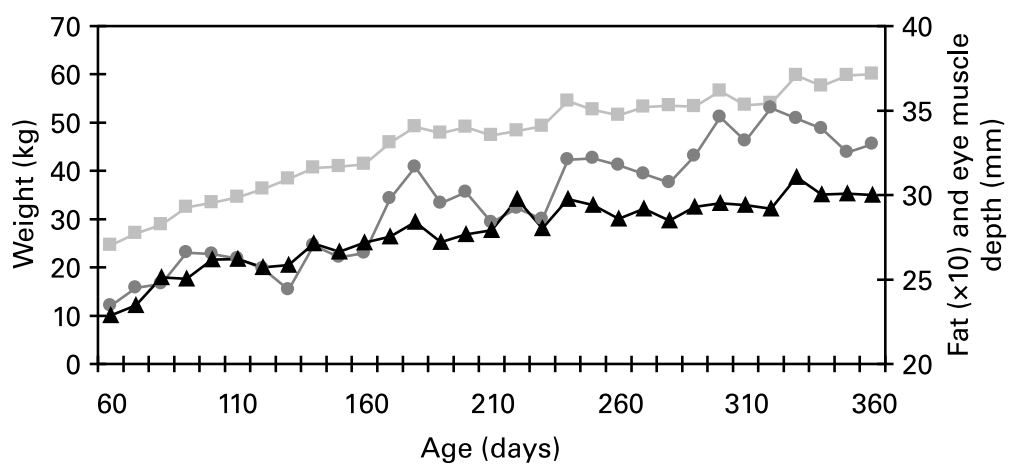

Figure 2 Mean weight (squares), fat depth (circles) and eye muscle depth (triangles) for individual ages in 10-day intervals.

\section{Results and discussion}

Means over time

Figure 2 shows the corresponding mean for BW, FD and EMD measures for individual ages in 10-day intervals. It shows that growth was almost linear until around 180 days, which coincides with commencement of maturity (onset of fat). Growth slowed over the rest of the trajectory. A similar pattern appeared in the data used by Fischer et al. (2004), and mean values corresponded well with other Poll Dorset data at similar ages. Figure 2 also shows how measures of FD increased throughout the 300-day trajectory, with a sharper increase relative to BW and EMD around 200 days of age, which coincided with the slowing of growth. Similarly, EMD increased steadily with age, but it slowed beyond 200 days. Mean values for the three traits were all considered representative of industry data (D. Brown, personal communication, 2003). Furthermore, means obtained for FD and EMD measures were consistent with other Poll Dorset data sets at similar ages (Gilmour et al., 1994; van der Werf and Wheaton, 1999).

\section{Random regression coefficients}

The covariance matrix between random regression coefficients (K) was estimated for each random effect using ASREML (Gilmour et al., 2002). A canonical decomposition of $\mathbf{K}$ into EDE' showed that the first eigenvalue in $\mathbf{D}$, dominated by the intercept, explained a large proportion of the variance $(0.85$ to 0.90$)$, as seen in Table 2 . Plotting the values in $\boldsymbol{\Phi E}$ from each analysis showed that the first eigenfunction was flat, which implies that the major part of the variation in BW, FD or EMD with age was explained by a factor that was constant for all ages. Selection on the first principal component would increase each trait at all ages.

However, an interesting pattern was seen in the second eigenfunction for each trait (not shown). Selection on a second canonical variable for BW would decrease weight at early ages and increase BW at late ages, and therefore selection on this variable would result in a change of the growth curve. In addition, while the majority of variation for FD and EMD was expressed in a factor that was constant for all ages, there is still scope to change fat and muscle deposition at different ages through selection on the second eigenfunction.
Estimates of variance, of the RR coefficients for each random effect from the BW analysis (not shown) were higher than those found by Fischer et al. (2004), which is directly related to the level of phenotypic variance which was higher in this data set. The relative magnitude of matrices estimated for the different random effects in this study were similar to those of Fischer et al. (2004), suggesting that the partitioning of variance over the different random effects was similar in both studies, even though the data sets were completely different.

\section{Covariance functions}

Estimates of variance for BW as a function of age are shown in Figure 3 using a quadratic RR model for direct genetic and environmental effects and a linear RR model for maternal genetic effects. Additive genetic variance remained relatively steady over the 300-day trajectory in this study, with values of 6,12 and $13 \mathrm{~kg}^{2}$ at 120,210 and 360 days. Comparison with genetic variance estimates from Fogarty (1995), which were 5, 10 and $17 \mathrm{~kg}^{2}$ for 100,200 and 365 days, showed a smaller increase of genetic variance at later ages ( $>250$ days) in our study. This small increase coincided with a sharp increase in permanent environmental variance at those ages, which is explained by a strong $(<-0.74)$ sampling correlation that exists between these the two direct effects at all ages. This pattern of partitioning has also been shown to be a problem in RR analysis of beef cattle growth data (Meyer, 2002 and 2003). Maternal genetic variance was shown to be relatively constant throughout the trajectory. Phenotypic variance increased steadily with age in this data set, with values of 30,42 and $66 \mathrm{~kg}^{2}$ at 120,210 and 360 days respectively. These agree closely with the values of 25,45 and $68 \mathrm{~kg}^{2}$ presented by Fogarty (1995) at 100, 200 and 365 days.

Variance components for FD and EMD are shown in Figures 4 and 5 .

Table 2 Percentage of genetic variation explained by each eigenvalue for weight, fat and muscle analyses

\begin{tabular}{lrcc}
\hline \hline Eigenvalue & Weight & Fat depth & Muscle depth \\
\hline First & 90 & 85 & 90 \\
Second & 6 & 15 & 10 \\
Third & 4 & & \\
\hline \hline
\end{tabular}




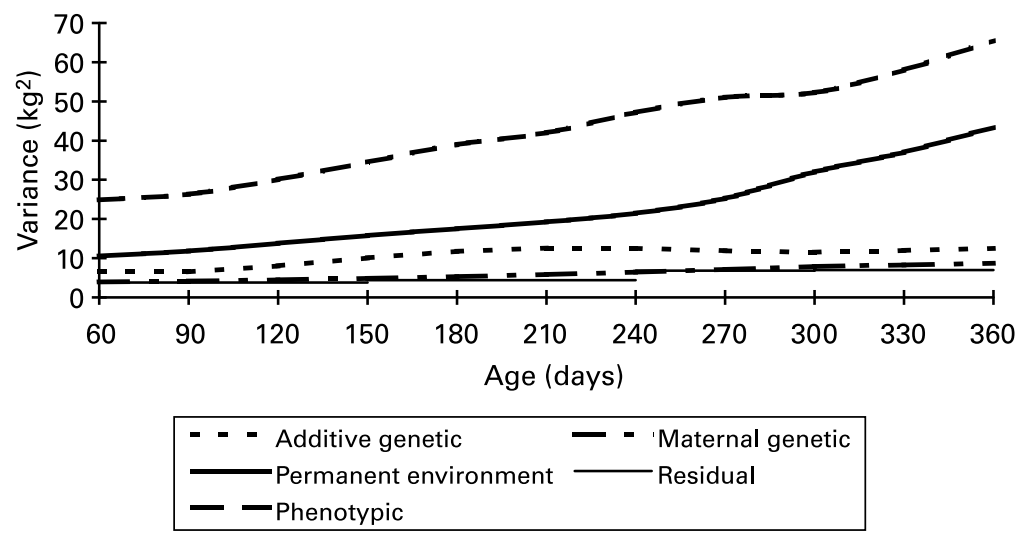

Figure 3 Variance component estimates for weight as a function of age in Poll Dorset sheep.

Additive genetic variance for FD was relatively constant throughout the trajectory at around $0.2 \mathrm{~mm}^{2}$ (Figure 4). Phenotypic variance was relatively constant at 0.4 to $0.5 \mathrm{~mm}^{2}$ but increased to $0.7 \mathrm{~mm}^{2}$ towards the end of the trajectory, which corresponds to the ages where animals approach maturity and hence deposit more fat. An age corrected analysis of FD by Maniatis and Pollott (2002) yielded estimates of 0.16 and $0.6 \mathrm{~mm}^{2}$ for genetic and phenotypic variance in Suffolk sheep at 150 days, which correspond reasonably well with estimates at similar ages in this study. Interestingly, our estimates of genetic and permanent environmental variance were slightly higher at younger ages $(<90$ days), which may result from the use of polynomials and fewer data at these ages (Figure 1).

Estimates of variance components for EMD shown in Figure 5 were similar to the FD results; additive genetic variance was relatively constant throughout the trajectory at around $2 \mathrm{~mm}^{2}$. Phenotypic variance started around $8 \mathrm{~mm}^{2}$, decreased to $6 \mathrm{~mm}^{2}$ at around 200 days and then increased to around $8 \mathrm{~mm}^{2}$ towards the end of the trajectory. From an age corrected analysis of eye-muscle depth, Maniatis and Pollott (2002) presented estimates of 1.7 and $7.2 \mathrm{~mm}^{2}$ for genetic and phenotypic variance in Suffolk sheep at 150 days, which correspond very well with estimates at similar ages in this study. Again, variances were slightly higher at younger ages $(<120$ days).

\section{Heritability}

Estimates of heritability from the analysis of BW, FD and EMD measures are given in Figure 6. Direct heritability for BW ranged from 0.24 to 0.30 for much of the trajectory but declined, unexpectedly, late in the trajectory ( $>240$ days). This coincided with an increase in permanent environmental variance as a proportion of phenotypic variance. Fogarty (1995) found heritability estimates of $0.20,0.23$ and 0.25 at 100,200 and 365 days, whereas the values in this study were $0.27,0.30$ and 0.20 at these ages.

For FD, heritability was reasonably constant, with values declining from 0.35 to 0.25 across the trajectory (Figure 6). Comparison with other studies involving Poll Dorset sheep included van der Werf and Wheaton (1999), who presented weight corrected estimates of 0.16 and 0.31 for FD at 200 and 365 days respectively, while Gilmour et al. (1994) produced weight corrected heritabilities of 0.12 and 0.33 at similar ages. Atkins et al. (1991) showed age corrected estimates of $0.28,0.26$ and 0.31 and weight corrected estimates of $0.22,0.32$ and 0.33 at 150,270 and 400 days of age respectively. McEwan et al. (1993) presented heritabilities for ultrasonic fat depth at the $\mathrm{C}$ site in Romneys of $0.31,0.45,0.29$ and 0.28 at $5,6,8$ and 14 months respectively which correspond reasonably well with our estimates with the exception of the 6 month estimate.

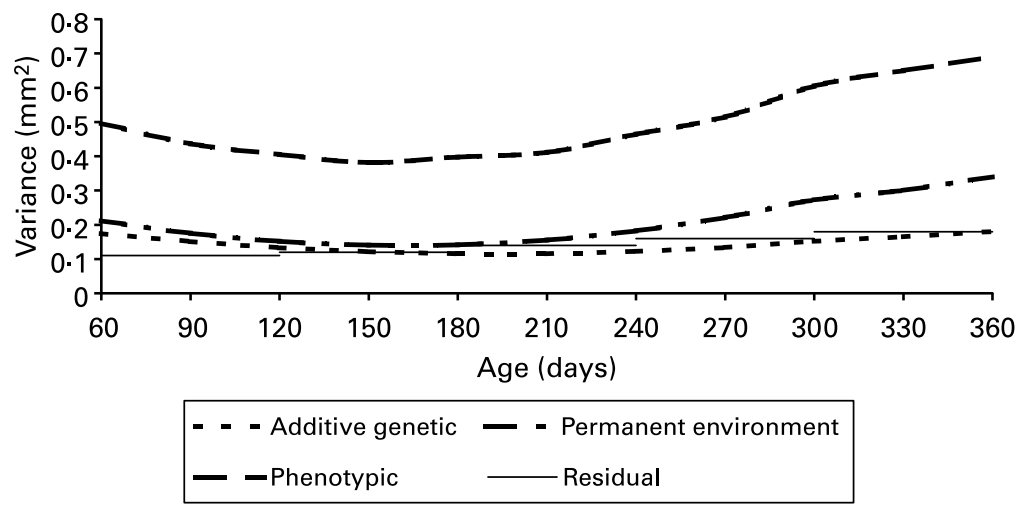

Figure 4 Variance component estimates for fat depth as a function of age in Poll Dorset sheep. 
Fischer, van der Werf, Banks, Ball and Gilmour

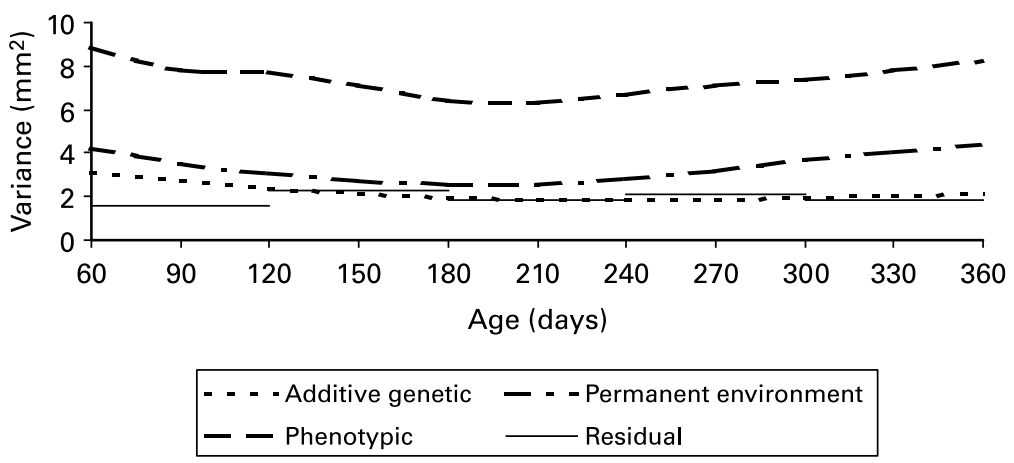

Figure 5 Variance component estimates for eye muscle depth as a function of age in Poll Dorset sheep.

Eye-muscle depth heritability estimates were similar to the fat depth estimates. The heritability across ages dropped from 0.35 to 0.25 over the 300-day trajectory (Figure 6). Van der Werf and Wheaton (1999) presented weight corrected estimates of 0.32 and 0.27 for muscle depth at 200 and 365 days respectively in Poll Dorsets.

\section{Correlations within traits at selected ages}

Table 3 shows the phenotypic and genetic correlations between BW at selected ages. Patterns were similar for genetic and phenotypic correlations in that they both decreased as age increased. Of interest is that the genetic correlation between early (day 60) and late (day 360) weight was considerably less than unity $(0.60)$, suggesting that early weights are not under the same genetic control as weights taken at older ages. This has implications for the potential to select for animals with different growth patterns. Lewis and Brotherstone (2002) reported a similar pattern to that seen in Table 3, as did Fischer et al. (2004), albeit with a lower correlation (0.39) between weights taken at the extreme ages, which may be due to more sparse data at the extremes in their data.

Maternal genetic correlations (not shown) were high $(>0.80)$ for all ages, which is consistent with the proposition that, although genes have their influence at early ages, the effects carry over to later ages of the trajectory. This was also found by Fischer et al. (2004). Table 4 shows correlations between FD at selected ages. The pattern of genetic and phenotypic correlations was similar to results of McEwan et al. (1993), although phenotypic correlations declined more rapidly in our study.

Table 5 shows the correlations between age corrected eyemuscle depths at selected ages. The genetic correlation between early (day 60) and late (day 360 ) measures of eyemuscle depth was 0.50 .

A genuine absence of literature estimates of genetic and phenotypic correlations between carcass measures at different ages make comparisons limited. Fogarty (1995) presented a genetic correlation of 0.70 between FD measures taken at 200 and 365 days of age. The work of Beatson (1987) using Coopworths supports this, with an estimated genetic correlation of 0.69 between weight corrected FD taken at 210 and 390 days of age. These estimates are lower than our estimates between 210 and 360 days of age, which were 0.86 and 0.93 from age and weight corrected analyses, respectively. Furthermore, Fogarty (1995) presented a genetic correlation of 0.75 between eye-muscle depths taken at 200 and 365 days of age, which is lower than our estimates at similar ages; 0.87 and 0.97 from the age and weight corrected analyses, respectively.

Variance estimates, heritabilities and genetic correlations between repeated measures of the same trait obtained from bivariate $R R$ analysis were very similar to those just

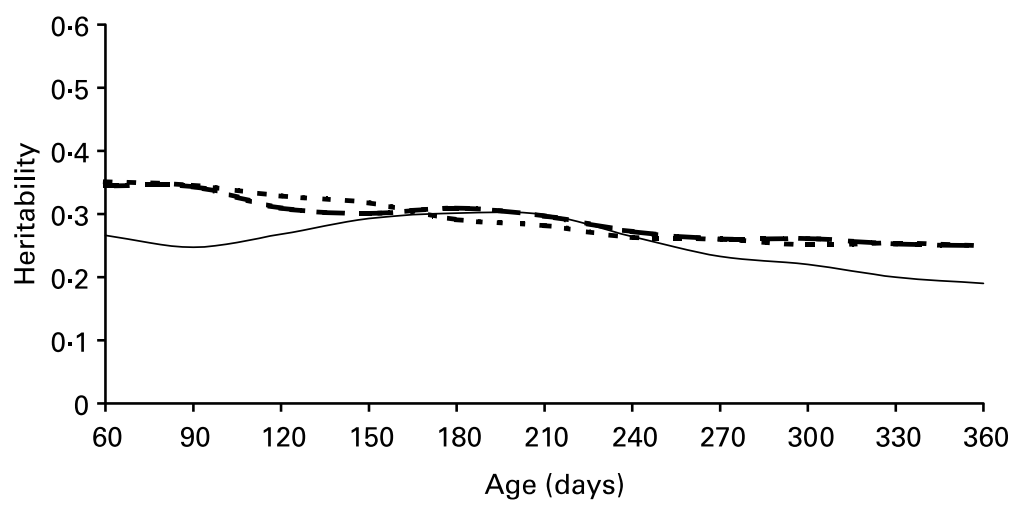

Figure 6 Estimates of direct heritability across age for weight (solid line), fat (dotted line) and eye muscle depth (dashed line) analyses (age corrected). 
Weight, fat and muscle depth in growing lambs

Table 3 Genetic (below diagonal) and phenotypic (above diagonal) correlations between weight at selected ages (in days)

\begin{tabular}{lllllll}
\hline \hline Age & 60 & 120 & 180 & 240 & 300 & 360 \\
\hline 60 & 1 & 0.75 & 0.65 & 0.58 & 0.56 & 0.50 \\
120 & 0.74 & 1 & 0.85 & 0.78 & 0.67 & 0.54 \\
180 & 0.54 & 0.96 & 1 & 0.84 & 0.75 & 0.62 \\
240 & 0.51 & 0.92 & 0.98 & 1 & 0.85 & 0.76 \\
300 & 0.57 & 0.81 & 0.84 & 0.92 & 1 & 0.96 \\
360 & 0.60 & 0.65 & 0.65 & 0.77 & 0.96 & 1 \\
\hline \hline
\end{tabular}

presented, which were obtained from univariate RR models for BW, FD and EMD data and are not presented here.

\section{Phenotypic correlations between traits at various ages}

Phenotypic correlations presented between BW and EMD in Figure 7 were moderate and positive and relatively constant throughout, declining only very slightly between the start and the end of the trajectory. Phenotypic correlations between BW and EMD were higher at any set of ages than correlations between BW and FD, which is compatible with the pattern of growth, and muscle development shown in Figure 1, where means for BW and EMD increased similarly whereas at older ages FD increased more rapidly. Gilmour et al. (1994) presented correlations of 0.51 and 0.62 at average ages of 7 and 12 months respectively, which are similar to our estimates of 0.65 at 210 and 360 days.

Phenotypic correlations between BW and FD are also presented in Figure 7. Between measures of the two traits at the same age, the correlation declined very slowly throughout the trajectory, from 0.58 to 0.51 . Interestingly, the phenotypic correlations between BW and FD, and FD and EMD were very similar (Figure 7). Again this can be related back to the trends shown in Figure 1, where FD increased faster relative to BW and EMD. Similar results were found by Atkins et al. (1991) who reported phenotypic correlations of $0.58,0.57$ and 0.48 between measures of age corrected BW and FD at average ages of 5, 9 and 14 months respectively. Furthermore, McEwan et al. (1993) presented correlations of $0.47,0.46,0.47$ and 0.52 between BW and FD at ages of 5, 6, 8 and 14 months. Estimates from these two studies correspond well with estimates presented in this study $(0.53,0.48$ and 0.51 at 150,270 and 360 days of age). Also, Brash et al. (1992) reported correlations of 0.52 and 0.51 in Border Leicester data at average ages of 290 and 420 days.

Table 4 Genetic (below diagonal) and phenotypic (above diagonal) correlations between fat depths at selected ages (in days)

\begin{tabular}{lllllll}
\hline \hline Age & 60 & 120 & 180 & 240 & 300 & 360 \\
\hline 60 & 1 & 0.71 & 0.56 & 0.37 & 0.20 & 0.13 \\
120 & 0.97 & 1 & 0.63 & 0.51 & 0.38 & 0.32 \\
180 & 0.85 & 0.95 & 1 & 0.61 & 0.54 & 0.51 \\
240 & 0.65 & 0.82 & 0.95 & 1 & 0.65 & 0.64 \\
300 & 0.42 & 0.63 & 0.83 & 0.96 & 1 & 0.72 \\
360 & 0.31 & 0.55 & 0.76 & 0.93 & 0.99 & 1 \\
\hline \hline
\end{tabular}

Table 5 Genetic (below diagonal) and phenotypic (above diagonal) correlations between eye muscle depths at selected ages (in days)

\begin{tabular}{lllllll}
\hline \hline Age & 60 & 120 & 180 & 240 & 300 & 360 \\
\hline 60 & 1 & 0.74 & 0.66 & 0.49 & 0.33 & 0.24 \\
120 & 0.98 & 1 & 0.68 & 0.57 & 0.45 & 0.38 \\
180 & 0.92 & 0.97 & 1 & 0.66 & 0.60 & 0.55 \\
240 & 0.79 & 0.88 & 0.97 & 1 & 0.69 & 0.67 \\
300 & 0.60 & 0.73 & 0.87 & 0.97 & 1 & 0.76 \\
360 & 0.50 & 0.65 & 0.80 & 0.93 & 0.99 & 1 \\
\hline \hline
\end{tabular}

Phenotypic correlations between FD and EMD measures shown in Figure 7 displayed a similar pattern to that of the genetic correlations between these two traits, in that phenotypic correlations were lower at older ages $(>0.55$ for ages below 120 days and around 0.4 for ages above 180 days). Gilmour et al. (1994) presented phenotypic correlations of 0.42 and 0.57 between measures of age corrected FD and EMD at average ages of 7 and 12 months, compared with estimates of 0.45 and 0.50 at 210 and 360 days in our study. Other literature estimates show moderate positive phenotypic correlations between these traits at all ages reported (Fogarty, 1995).

\section{Genetic correlations between traits at various ages}

Genetic correlations between BW and FD are presented in Figure 8. The correlation between measures for the two traits at the same age declined slowly from 0.73 to 0.67 , demonstrating that the correlation between measures of BW and FD at various ages is quite similar. However, the correlation between successive measures of weight and fat were sufficiently different from one to suggest that animals can be genetically heavy and lean at any age. Given the pattern of growth and fat development illustrated in Figure 1, where fat appeared to increase more relative to weight beyond 180 days, one might expect the genetic correlation between these two traits to decline at these older ages, but this was not the case.

Using Poll Dorset data, Atkins et al. (1991) estimated a genetic correlation of 0.72 between BW and FD measures at an average age of 5 months which corresponds well with our estimate of 0.71 at 150 days. However, the correlations between measures at an average age of 9 months and 14 months were 0.44 and 0.42 in the study of Atkins et al. (1991), which is significantly lower than our estimates at 270 and 360 days $(0.66$ and 0.67 , respectively). Furthermore, McEwan et al. (1993) presented genetic correlations in Romney sheep of $0.56,0.45,0.38$ and 0.67 between BW and FD at ages of 5, 6, 8 and 14 months. Brash et al. (1992) estimated a genetic correlation of 0.77 between weight and fat measures at an average age of 290 days in Border Leicester data, which is more in agreement with our results. Despite minor differences, the correlation pattern was similar for all three studies, in that correlations were moderate to high and positive, which is supported by our results.

Genetic correlations between BW and EMD shown in Figure 8 declined slightly from 0.71 to 0.63 for measures of BW and EMD at the same age throughout the trajectory. 


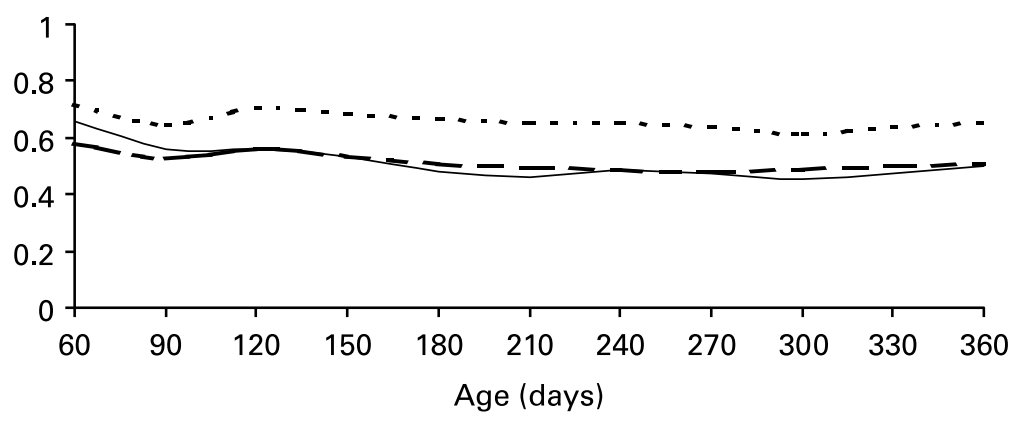

Figure 7 Phenotypic correlations between traits over time: fat and muscle (solid line), weight and muscle (dotted line) and weight and fat (dashed line).

Correlations between age corrected BW and EMD (Figure 8) were lower but showed a similar pattern to those found by Gilmour et al., (1994), which were $0.48,0.46$ and 0.41 at average ages of 7,12 and 16 months.

Correlations between measures of FD and EMD shown in Figure 8 declined with age of measurement, from 0.77 between measures of FD and EMD at 60 days to 0.43 at 300 days of age. This suggests that there is scope for selecting animals to be lean and muscular, in particular at later ages due to the lower correlation. This decline in correlation with age is consistent given the trait average curves (Figure 1) where FD relative to EMD increased at later ages (>200 days). Gilmour et al. (1994) presented correlation estimates of 0.57 and 0.58 between measures of age corrected FD and EMD at 12 and 16 months.

\section{General discussion}

Models used. This study was primarily focussed on patterns of variation in the three traits considered, rather than statistically comparing different models. Fischer et al. (2004) used statistical criteria to determine that for weight data a quadratic polynomial for direct genetic and environmental and maternal environmental effects and a linear polynomial for maternal genetic effects gave the best fit. Fischer et al. (2004) encountered variance partitioning problems between maternal effects when fitting both genetic and environmental components and in order to circumvent this problem, it was decided to fit maternal genetic effects only. Meyer (1992) showed in her study that fitting only one maternal component invariably accommodates most of the total variation due to all maternal effects, however it should be noted that this approach will produce an inflated estimate of maternal genetic effects because environmental variance is included in the estimate.

Considering that the data structure used in this study was similar to that used by Fischer et al. (2004) and the average number of records per animal in the weight data of this study was also similar $(2.7$ v. 3.1), we suggested that a quadratic polynomial could be fitted for each effect. However, the maternal genetic component of the weight analysis was fitted using a linear polynomial, as no significant increase in the Log Likelihood was seen when adding the quadratic component. Similarly, a linear model was fitted for direct genetic and environmental effects in the fat and muscle analyses, as the average number of records per animal was $1 \cdot 8$

Genetic parameters. Description of how weight, fat and muscle change with age is of interest and important to prime lamb producers. The lack of published information about how carcass traits change with age and their relationships throughout life has been partly filled by this research. This is the first report on growing lambs, and probably even on livestock in general, with a number of repeated measures of weight as well as muscle and fat depth, and therefore the first time that such a relationship could be studied over the development trajectory. An alternative method for determining carcass composition at various ages is computer tomography (CT) scanning (e.g. Banks, (1997);

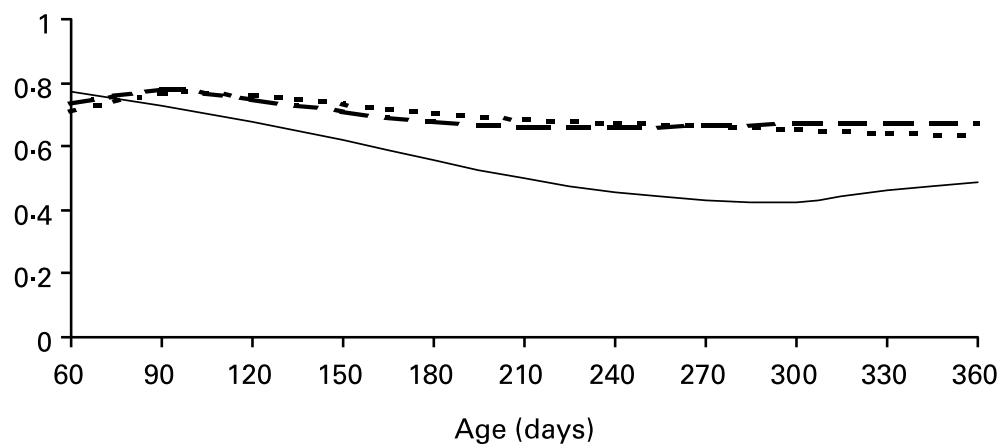

Figure 8 Genetic correlations between traits over time: fat and muscle (solid line), weight and muscle (dotted line) and weight and fat (dashed line). 
Jopson et al., (1997); Lewis et al. (2004)), however, given both the expense and lack of portability of this technology it is unlikely to be of major use in extensively managed industries such as the Australian lamb industry.

Heritabilities and genetic correlations found in this study were generally in agreement with literature estimates at specific ages. In particular, the correlation pattern between repeated measures of the same trait (correlations decline as age between measures increases) suggests that animals differ in their genetic propensity to grow, deposit fat and develop muscle at different ages throughout life. It was also shown that animals with genes for faster growth tend to be more muscular and fatter and that the relationships between these traits are reasonably constant, with only some loosening of the relationship of weight, fat and muscle as animals grow toward maturity.

However, the correlations were sufficiently different from unity to suggest that animals can be heavy, lean and muscular at the same time and at different ages. Banks and Ross (2003) suggested that such lambs are more profitable for producers due to more efficient production, higher meat yields and more valuable carcasses. However, it is likely that producers may only want to change carcass traits in one direction (e.g. decrease fat depth, increase muscle) throughout the whole life of the animal. Their aim will be to achieve a certain weight and fatness at a particular age with growth path efficiency considerations also of importance.

The parameters estimated in this paper also provide insight into the optimal ages of recording for the individual traits. For weight, measures are required at 100 days to give an indication of early growth (direct and maternal), 200 days to correlate to carcass measures and 365 days to give an indication of mature size. If breeders were to measure carcass traits only once, then the recommendation would be for this to take place at around 200 days of age. Measures earlier than this do not give a good indication of genetic potential at later ages (genetic correlation $<0.50$ ) whereas measurement at 200 days gives a better indication of performance at 360 days (genetic correlations close to 0.80 ), which has relevance for markets requiring heavier lambs. These ages are the ages at which LAMBPLAN currently recommend that breeders collect their data (Brown et al., 2000).

Given that the RR method provides genetic information within and between traits at all ages in the trajectory, different emphasis could be placed in an index using information from different ages. Again, this is based on the underlying correlation structure that exists throughout time between repeated measures of these three traits. For example, one could envisage placing low emphasis on FD at young ages as animals have not yet reached a stage of maturity (e.g. $<180$ days), and high emphasis on decreasing FD at older ages (>180 days) to select genetically leaner animals to meet market specifications.

Age or weight corrected carcass traits. The arguments surrounding the correction of carcass measures for age or weight remain. When correcting one trait (e.g. muscle) using another trait (e.g. weight) as a covariate, the correlations between them are difficult to interpret and the phenotypic correlation has an expectation close to zero. It can be shown that selection for traits corrected for other traits is equivalent to selection for the two traits simultaneously when considering their correction (Kennedy et al., 1993). However, with fixed effects, differences may occur as the trait used as a covariable is often not appropriately corrected for fixed effects. Furthermore, with phenotypic correction, it is not immediately evident how much genetic covariation is also being corrected for. Therefore defining conditional traits after phenotypic and genetic relationships have been established seems a more appropriate approach. Furthermore, it is important to have an appropriate breeding objective defined, where change in profit after multiple trait selection is not double counted across traits.

Considering a biological rather than statistical perspective, Berg and Butterfield (1976) suggested that fat and muscle development is influenced to a greater extent by body weight, than by age. Thompson and Butterfield (1985) showed that stage of maturity, which is highly correlated to body weight, was the major driving force behind development of fat and muscle in sheep growth. In light of this, additional analysis of fat and muscle was performed using weight as a covariate instead of age (results not shown). The weight corrected analyses had lower phenotypic variance, but weight correction did not affect variance partitioning or estimates of heritability, which is supported by the review by Fogarty (1995). McEwan et al. (1991) also showed only slight differences between genetic parameter estimates of carcass traits from age or weight corrected analyses.

\section{Conclusions}

This study presented genetic and phenotypic relationships between repeated measures of weight, fat and muscle depth at different ages in a population of Australian sheep. In doing so, this study highlights the potential to select on weight, fat and muscle trajectories, as well as the scope for change of each trait at different ages through the correlations. This information is of immense value to the industry and will enable breeders to optimise selection for specific combinations of weight, fat and muscle attributes at particular ages to suit specific objectives. It can be concluded that random regression models could be used in routine analysis of sheep carcass measures, however, an increase in the current level of recording would be recommended to allow more precise breeding value estimation.

\section{Acknowledgements}

The financial support of Meat and Livestock Australia towards this research is gratefully acknowledged as well as the additional funding for data collection. The breeders involved in the Meat Elite program are thanked for their involvement in the collection and submission of data. Stefan Spiker, Stephen Field and Matt Kelly are gratefully acknowledged for their contribution towards collection, submission and preparation of data.

\section{References}

Atkins, K. D., Murray, J. I., Gilmour, A. R. and Luff, A. L. 1991. Genetic variation in live weight and ultrasonic fat depth in Australian 
Poll Dorset sheep. Australian Journal of Agricultural Research 42: 629-640.

Banks, R. G. 1990. Lambplan: An integrated approach to genetic improvement for the Australian lamb industry. Proceedings of the Association for the Advancement of Animal Breeding and Genetics 8: 237-240.

Banks, R. G. 1997. The Meat Elite Project: Establishment and achievements of an elite meat sheep nucleus. Proceedings of the Association for the Advancement of Animal Breeding and Genetics, Dubbo, Australia, 12: pp. 598-601.

Banks, R. G. and Ross, I. S. 2003. Information flow in lamb supply chains-implications for terminal sire breeding. Proceedings of Association for the Advancement of Animal Breeding and Genetics, Melbourne, Australia, 15: pp. 334-337.

Beatson, P. R. 1987. The inheritance of live weight-corrected fatdepth in Coopworth ram hoggets. Proceedings of Association for the Advancement of Animal Breeding and Genetics, Perth, Australia, 6: pp. 87-90.

Berg, R. T. and Butterfield, R. M. 1976. New concepts of cattle growth. Macarthur Press, Sydney.

Brash, L. D., Fogarty, N. M., Gilmour, A. R. and Luff, A. F. 1992. Genetic parameters for liveweight and ultrasonic fat depth in Australian mean and dual-purpose sheep breeds. Australian Journal of Agricultural Research 43: 831-841.

Brown, D. J., Tier, B., Reverter, A., Banks, R. and Graser, H. U. 2000. OVIS: A multiple trait breeding value estimation program for genetic evaluation of sheep. Wool Technology and Sheep Breeding 48: $285-297$.

Fischer, T. M., Werf, J. H. J., van der, Banks R. G. and Ball, A. J. 2004. Description of lamb growth using random regression on field data. Livestock Production Science 89: 175-185.

Fogarty, N. M. 1995. Genetic parameters for live weight, fat and muscle measurements, wool production and reproduction in sheep: a review. Animal Breeding Abstracts 63: 101-143.

Gilmour, A. R., Cullis B. R., Welham, S. J. and Thompson, R. 2002. ASREML reference manual. NSW Agriculture, Orange, Australia.

Gilmour, A. R., Luff, A. F., Fogarty, N. M. and Banks, R. G. 1994. Genetic parameters for ultrasound fat depth and eye muscle measurements in live Poll Dorset sheep. Australian Journal of Agricultural Research 45: 1281-1291.

Gilmour, A. R., Thompson, R. and Cullis, B. R. 1995. Al, an efficient algorithm for REML estimation in linear mixed models. Biometrics 43: 277-288.

Hassen, A., Wilson, D. E. and Rouse, G. H. 2003. Estimation of genetic parameters for ultrasound-predicted percentage of intramuscular fat in Angus cattle using random regression models. Animal Science 81: $35-45$.

Huisman, A. E. 2002. Genetic analysis of growth and feed intake patterns in pigs. Ph.D. thesis, Wageningen Institute of Animal Sciences, Wageningen.

Huisman, A. E., Veerkamp, R. F. and Arendonk, J. A. M. van. 2002. Genetic parameters for various random regression models to describe the weight data of pigs. Animal Science 80: 575-582.

Jopson, N. B., McEwan, J. C., Fennessey, P. F., Dodds, K. G., Nicoll, G. B. and Wade, C. M. 1997. Economic benefit of including computed tomography measurements in a large terminal sire breeding program. Proceedings of the Association for the Advancement of Animal Breeding and Genetics 12: 72-75.

Kennedy, B. W., Werf, J. H. J. van der and Meuwissen, T. H. E. 1993. Statistical and genetic properties of residual feed intake. Journal of Animal Science 71: 3239-3250.

Kinghorn, B. P. 1997. Genetic improvement in sheep. In The genetics of sheep (ed. A. Ruvinsky), pp. 565-591, CAB International: Armidale, Australia.

Kirkpatrick, M., Lofsfold, D. and Bulmer, M. 1990. Analysis of the inheritance, selection and evolution of growth trajectories. Genetics 124: $979-993$

Lewis, R. M. and Brotherstone, S. 2002. A genetic evaluation of growth in sheep using random regression techniques. Animal Science 74: 63-70.

Lewis, R. M., Macfarlane, J. M., Simm, G. and Emmans, G. C 2004. Effects of food quality on growth and carcass composition in lambs of two breeds and their cross. Animal Science 78: 355-367.

McEwan, J. C., Clarke, J. N., Hickey, S. M. and Knowler, K. J. 1993. Heritability of ultrasonic fat and muscle depths in Romney sheep. Proceedings of the New Zealand Society of Animal Production, Hamilton, New Zealand 53: pp. 347-350.

McEwan, J. C., Dodds, K. G., Davis, G. H., Fennessey, P. F. and Hishon, M. 1991. Heritability of ultrasonic fat and muscle depths in sheep and their correlations with production traits. Proceedings of the Association for the Advancement of Animal Breeding and Genetics, Melbourne, Australia 9: pp. 276-279.

Maniatis, N. and Pollott, G. E. 2002. Maternal effects on weight and ultrasonically measured traits of lambs in a small closed Suffolk flock. Small Ruminant Research 45: 235-246.

Meyer, K. 1992. Variance components due to direct and maternal effects for growth traits of Australian beef cattle. Livestock Production Science 31: 179-204.

Meyer, K. 2002. Estimates of covariance functions for growth of Australian beef cattle from a large set of field data. Proceedings of seventh world congress genetics applied to livestock production Montpellier, France, CD-ROM communication no. 11-01

Meyer, K. 2003. First estimates of covariance functions for lifetime growth of Angus cattle. Proceedings Association for Advancement of Animal Breeding and Genetics, Melbourne, Australia 15: pp. 395-398.

Statistical Analysis Systems Institute, 1988. SAS/STAT user's guide, release 6.03 edition. SAS Institute, Cary, NC.

Thompson, J. M. and Butterfield, R. M. 1985. Food intake, growth and body composition in Australian Merino sheep selected for high and low weaning weight 2 . Chemical and dissectible body composition. Animal Production 40: 71-84.

Veerkamp, R. F. and Thompson, R. 1999. A covariance function for feed intake, live weight, and milk yield estimated using a random regression model. Journal of Dairy Science 82: 1565-1573.

Werf, J. H. J. van der and Wheaton, T. 1999. Estimation of genetic parameters for live weight, fat and muscle measurements in seven sheep breeds based on LAMBPLAN data. MLA Report, Sydney, Australia, 165 Walker St, North Sydney, Australia.

(Received 18 February 2005-Accepted 26 August 2005) 